Full length article

\title{
Fast-dissolving electrospun nanofibrous films of paracetamol/cyclodextrin inclusion complexes
}

\author{
Zehra Irem Yildiz ${ }^{\mathrm{a}}$, Tamer Uyar ${ }^{\mathrm{a}, \mathrm{b}, *}$ \\ ${ }^{a}$ Institute of Materials Science \& Nanotechnology, Bilkent University, Ankara 06800, Turkey \\ ${ }^{\mathrm{b}}$ Department of Fiber Science \& Apparel Design, College of Human Ecology, Cornell University, Ithaca, NY 14853, USA
}

\section{A R T I C L E I N F O}

\section{Keywords:}

Paracetamol

Cyclodextrin inclusion complexes

Electrospinning

Nanofibers

Fast-dissolving

\begin{abstract}
A B S T R A C T
The free-standing and fast-dissolving nanofibrous films from inclusion complexes (ICs) of paracetamol with two different cyclodextrins, hydroxypropyl-beta-cyclodextrin (HP $\beta C D$ ) and sulfobutylether-beta-cyclodextrin (SBE$\beta$-CD) were produced through electrospinning without using polymer matrix. The morphology of the nanofibers (NFs) was uniform and bead-free as confirmed by scanning electron microscopy imaging. The chemical, structural and thermal characteristics of the electrospun paracetamol/CD-IC NFs were investigated by X-ray diffractometry, Fourier transform infrared spectroscopy, differential scanning calorimetry, thermal gravimetric analyzer and proton nuclear magnetic resonance. The aforementioned methods indicated the successful formation of ICs of paracetamol with both CD types (HP $\beta C D$ and SBE- $\beta-C D)$. Besides, paracetamol/CD-IC NFs exhibited different features and properties from pristine paracetamol. For instance, the crystalline state of pristine paracetamol was transformed into amorphous state by CD-IC NFs formation which is important for the water-solubility increment of the drug molecules. Moreover, thermal studies indicated that paracetamol became thermally more stable in CD-IC NFs. The molar ratio of paracetamol:CD was found as $\sim 0.85: 1.00$ for paracetamol/HP $\beta C D-I C$ NFs and $\sim 0.80: 1.00$ for paracetamol/SBE- $\beta$-CD-IC NFs. The dissolution behavior of paracetamol/CD-IC nanofibrous films was examined by exposing them to water. The electrospun paracetamol/CD-IC nanofibrous films showed fast-dissolving character in water due to the CD-ICs formation and high surface area of nanofibrous structure.
\end{abstract}

\section{Introduction}

The main challenge for the preparation of oral drug dosage formulations is due to poor bioavailability of drugs. Aqueous solubility, dissolution rate, drug permeability are the key factors affecting drug bioavailability. There are certain systems to improve the drug bioavailability and the use of cyclodextrins (CDs) is one of the promising alternative to improve aqueous solubility and dissolution rate of the drug molecules [1-4]. CDs are cyclic oligosaccharides having doughnut-shaped molecular structure (Fig. 1a) which are produced from enzymatic degradation of starch. The CDs have the ability to form noncovalent host-guest type inclusion complexes (ICs), thanks to their relatively hydrophobic inner cavity which is available for molecular encapsulation of drug molecules for drug delivery applications [1-4]. The formation of cyclodextrin inclusion complexes (CD-ICs) with drug molecules can provide certain advantages in drug delivery systems. The main advantages are increase in aqueous solubility, bioavailability and stability of drugs [1-4]. Besides, reduction and/or prevention of gastrointestinal, ocular irritation, and reduction or elimination of unpleasant taste and odor are the other advantages which CD-ICs systems can provide successfully [5]. CDs are of three native types referred to as $\alpha-C D, \beta-C D$, and $\gamma$-CDs that are composed of six, seven and eight glucopyranose units, respectively. These parent CDs can be modified by hydroxypropyl (HP), methyl (M) and sulfobutylether (SBE) groups in order to make them highly soluble in water which is quite crucial for drug delivery systems [6].

Electrospinning is a simple and cost-effective process to produce fibers with diameters ranging from nanometers to very few microns. The electrospun nanofibers are quite suitable for encapsulating drug substances since such nanofibrous matrix can provide distinctive properties such as easy drug entrapment, high surface area, nanoporous morphology [7]. Drug-loaded nanofibers provide controlled release and increase in dissolution rate of drugs $[8,9]$. Although the single nozzle electrospinning setup is very simple and versatile to produce functional nanofiber matrix incorporating drug molecules $[10,11]$, the use of advanced nozzle systems for the electrospinning of multiple-fluids are also

\footnotetext{
* Corresponding author at: Department of Fiber Science \& Apparel Design, College of Human Ecology, Cornell University, Ithaca, NY 14853, USA.

E-mail address: tu46@cornell.edu (T. Uyar).
} 


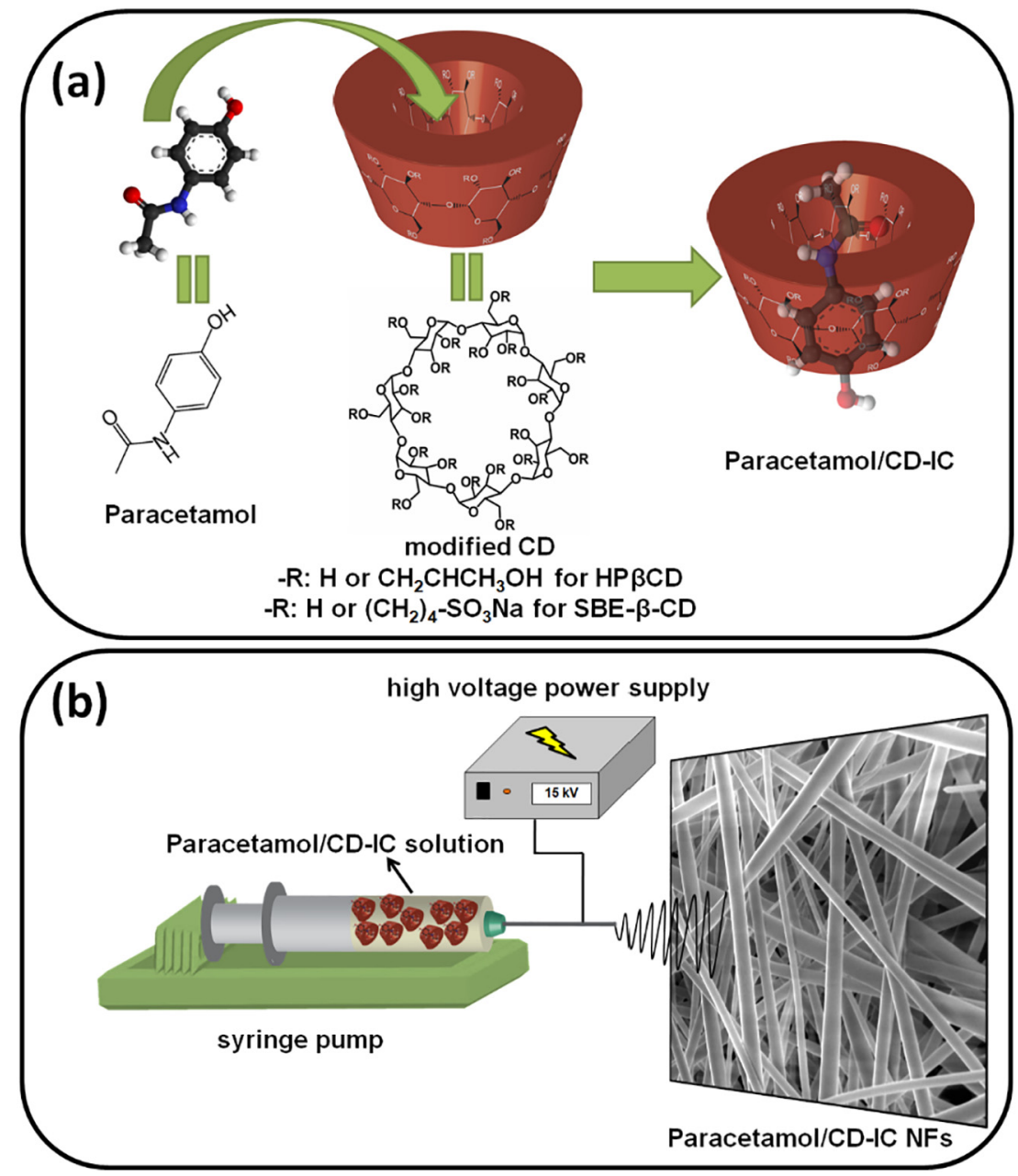

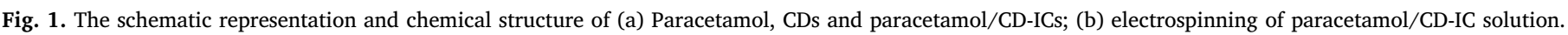

possible. For instance, coaxial electrospinning [12], side-by-side electrospinning [13] and tri-axial electrospinning [14] can be used for creating complex nanofibrous structures for drug delivery purposes. Electrospinning is also quite handy since this technique facilitates the production of nanofibers on a large scale $[15,16]$. In addition, it is possible to association the use of electrospinning technique with other traditional methods for drug delivery purposes [15] or electrospinning can be an alternative to freeze drying for drug formulations [16]. In general, electrospinning of nanofibers is performed by using polymeric solutions or polymer melts since overlapping and entanglement of polymer chains are very critical to get proper nanofiber production during the process [17]. Nevertheless, recently we have shown that, the electrospinning of non-polymeric systems (i.e. $\mathrm{CD}$ ) is possible to produce $C D$ nanofibers without the need of polymeric carrier matrix $[18,19]$. Moreover, electrospun nanofibers of CD-ICs with various guest molecules was also produced without using additional polymer matrix [20-25].

Paracetamol ( $N$-acetyl-para-aminophenol, also known as acetaminophen, Fig. 1a) is an antipyretic and analgesic agent which is used to relieve pain in the body and to reduce body temperature [26]. Although it has no significant anti-inflammatory activity compared to aspirin or ibuprofen, it is widely used since it is generally safe at recommended doses even for children and pregnant women [27]. There are studies in literature focusing on CD-ICs of paracetamol due to effects of CD-ICs on solubility, dissolution rate, absorption and chemical stability of drugs $[27,28]$. In one of those studies, it was shown that paracetamol can form stable ICs with native CDs; $\alpha$-CD, $\beta$-CD and $\gamma$-CD [28]. In another study, ICs of paracetamol with $\beta-C D$, hydroxypropyl- $\beta$ -
$\mathrm{CD}(\mathrm{HP} \beta \mathrm{CD})$ and methylated- $\beta-\mathrm{CD}(\mathrm{M} \beta \mathrm{CD})$ were prepared in $1: 1 \mathrm{M}$ ratio and the higher complexation stability with the two modified CDs (HP $\beta C D$ and $M \beta C D$ ) was observed when compared to native $\beta$-CD [27]. There are also studies in the literature related to encapsulation of paracetamol into hydrophilic polymeric electrospun nanofiber matrix to increase the water solubility and dissolution rate of paracetamol [26,29-32]. In a related study, the electrospun nanofiber based solid dispersion of paracetamol was prepared by using polyvinylpyrrolidone (PVP) as a hydrophilic fiber matrix and compared with conventional processes and the study showed that electrospun nanofibrous system has shown a better performance than conventional processes in terms of improving dissolution rate of paracetamol [29]. In another study, PVP nanofibers loaded with paracetamol and caffeine were fabricated by electrospinning and such hydrophilic nanofibrous matrix encapsulating paracetamol and caffeine has exhibited fast-dissolving character. However, in this system a flavoring agent was added to system in order to mask bitterness of drugs [26]. The literature shows that encapsulation of paracetamol by formation of CD-ICs and by electrospun nanofibers provide advantages on bioavailability of paracetamol especially in terms of its dissolution rate. However, in the literature, there is no example of electrospun nanofibers including CD-IC and the possible effects of this combination on bioavailability of paracetamol. Based on the literature and our previous experience, it is expected that formation of paracetamol/CD-IC nanofibers by using two modified CDs provides enhanced properties for paracetamol especially for its dissolving character in water.

The approach of electrospinning of nanofibers from hydrophilic polymers incorporating drug molecules are promising since drug 
molecules can be embedded in the nanofiber matrix as an amorphous form and such high surface area of hydrophilic polymeric nanofibrous materials incorporating drug molecules can lead to novel design of fastdissolving drug delivery systems [26,29,33-35]. In addition, the fastdissolving drug delivery systems based on CD-IC/drug incorporated hydrophilic electrospun nanofibers [10] or purely CD-IC/drug electrospun nanofibers without using any carrier polymeric matrix were also explored [24]. When drug molecules are complexed within the CD cavity, the drug molecules are separated from each other and hence cannot form crystalline domains in CD-ICs. Therefore, CD-ICs have positive effects on the solubility, dissolution rate and chemical stability of drug molecules. So, such electrospun nanofibers based on purely CDIC/drug systems can be very promising for the development of fastdissolving drug delivery systems. Even though electrospinning of nanofibers from purely CD-IC/drug systems are challenging when compared to electrospinning of polymeric systems, recently it has been shown that high-speed electrospinning with a novel continuous cyclone collection could manufacture a formulation of the poorly water-soluble antifungal voriconazole with sulfobutylether- $\beta$-cyclodextrin (SBE- $\beta$-CD) and this continuous process was suggested as alternative to freeze drying [16].

In this study, nanofibers (NFs) from CD-IC of paracetamol (paracetamol/CD-IC NFs) were produced via electrospinning. Here, paracetamol was separately complexed with two different modified CDs; HP $\beta C D$ and sulfobutylether- $\beta-C D$ (SBE- $\beta-C D$ ), and then paracetamol/ $\mathrm{CD}$-IC aqueous systems were electrospun into nanofibers without using additional polymeric matrix. The polymer-free electrospun paracetamol/CD-IC NFs were obtained as a free-standing webs having fastdissolving character in water.

\section{Materials and methods}

\subsection{Materials}

Paracetamol (99\%) was obtained from Sigma-Aldrich commercially. Sulfobutylether-beta-cyclodextrin (SBE- $\beta$-CD, Captisol ${ }^{\circledR}$ ) was kindly donated by Cydex Pharmaceuticals Inc. (Kansas, USA) and hydroxypropyl-beta-cyclodextrin (HP $\beta C D$ ) (Cavasol W7 HP Pharma) was donated by Wacker Chemie (Germany). Potassium bromide (KBr, $99 \%$, FTIR grade, Sigma-Aldrich), deuterated dimethylsulfoxide (d6-DMSO, deuteration degree min. $99.8 \%$ for NMR spectroscopy, Merck) were used as received. The water used was from a Millipore Milli-Q ultrapure water system.

\subsection{Preparation of paracetamol-cyclodextrin inclusion complexes (paracetamol/CD-IC) and electrospinning of paracetamol/CD-IC NFs}

Paracetamol was dispersed in $0.5 \mathrm{~mL}$ of water and then HP $\beta C D$ $(200 \%, \mathrm{w} / \mathrm{v})$ and SBE- $\beta-C D(240 \%, \mathrm{w} / \mathrm{v})$ was added to get $1: 1$ (paracetamol:CDs) molar ratio, respectively (Fig. 1a). The solutions were stirred at room temperature for $24 \mathrm{~h}$. For comparative studies, solutions for pure CDs were also prepared in water with concentration of $200 \%, w / v$ for $\mathrm{HP} \beta C D$ and $240 \%, w / v$ for SBE- $\beta-C D$.

The solutions of pure CDs and paracetamol/CD-ICs were separately loaded in $1 \mathrm{~mL}$ syringe having metallic needle of $0.4 \mathrm{~mm}$ inner diameter. These solutions were pumped through a syringe pump (KD Scientific, KDS-101, USA) at $0.5-1.0 \mathrm{~mL} / \mathrm{h}$ rate. Grounded metal covered with aluminum foil, a collector, was placed $15-20 \mathrm{~cm}$ from the tip of needle. High voltage at $15-20 \mathrm{kV}$ was applied between tip of needle and collector by high voltage power supply (Spellman, SL Series) (Fig. 1b).

\subsection{Measurements and characterization}

The viscosity of paracetamol/CD-IC solutions were measured by rheometer equipped with a cone/plate accessory (CP 20-4 spindle type, Anton Paar Physica MCR 301) under constant shear rate of $100 \mathrm{~s}^{-1}$ at RT. The Inolab $\mathrm{pH} /$ Cond 720-WTW was used to determine the conductivity of solutions at RT.

Scanning electron microscopy (SEM; Quanta 200 FEG; FEI) was used to perform morphological characterization of electrospun paracetamol/HP $\beta$ CD-IC NFs and paracetamol/SBE- $\beta$-CD-IC NFs. Au/Pd was sputtered on the nanofibers before SEM imaging to eliminate charging problems. Average fiber diameter (AFD) of nanofibrous films were calculated directly from SEM images by measuring the diameter of about 100 fibers.

The molar ratio between paracetamol and CDs was determined by using proton nuclear magnetic resonance $\left({ }^{1} \mathrm{H}\right.$ NMR; DPX-400, Bruker) spectra at $400 \mathrm{MHz}$ and $25^{\circ} \mathrm{C}$ by dissolving about $20 \mathrm{~g} / \mathrm{L}$ sample in DMSO- $d_{6}$.

Fourier transform infrared spectrometry (FTIR, Bruker-VER-TEX70) was used to obtain the infrared spectra of paracetamol, pure CD NFs and paracetamol/CD-IC NFs. The pellets of samples were prepared by mixing them with potassium bromide (KBr). The scans (64) were recorded between 4000 and $400 \mathrm{~cm}^{-1}$ at a resolution of $4 \mathrm{~cm}^{-1}$.

The crystalline structure of paracetamol, pure CD NFs and paracetamol/CD-IC NFs was recorded by X-ray diffractometry (XRD; X'Pert powder diffractometer; PANalytical) applying $\mathrm{Cu} K \alpha$ radiation in a $2 \theta$ range $5-30^{\circ}$.

Differential scanning calorimetry (DSC) (TA Q2000, USA) and thermogravimetric analysis (TGA) (TA Q500, USA) were used for the investigation of the thermal properties of the samples. DSC analyses were carried out under $\mathrm{N}_{2}$; initially, samples were equilibrated at $25^{\circ} \mathrm{C}$ and then heated to $200{ }^{\circ} \mathrm{C}$ at a heating rate of $20^{\circ} \mathrm{C} / \mathrm{min}$. TGA measurements were performed from room temperature to $600{ }^{\circ} \mathrm{C}$ at a heating rate of $20^{\circ} \mathrm{C} / \mathrm{min}$ under $\mathrm{N}_{2}$ atmosphere.

\subsection{Dissolution test}

The dissolution behavior of paracetamol/CD-IC nanofibrous films during water exposure was analyzed with two different ways. In the first one, nanofibrous films with equal weight were placed into petri dishes and $5 \mathrm{~mL}$ of distilled water was added onto the nanofibrous films. In the second one, absorbent paper was placed into petri dishes and thoroughly wetted with distilled water $(10 \mathrm{~mL})$. The excess water was completely drained out and the nanofibrous films were placed on the wet paper.

\section{Results and discussion}

\subsection{Morphological analyses of paracetamol/CD-IC NFs}

Electrospinning of paracetamol/CD-ICs was conducted under optimized parameters to obtain bead-free and uniform nanofibers. Besides optimization on electrospinning setup like voltage, distance between tip and collector, humidity and temperature, adjustment of solution concentration is quite essential for proper electrospinning process. In this study, high concentration of paracetamol/CD-ICs solutions was prepared for both $\mathrm{CDs}$ and bead-free and uniform nanofibers were obtained. The image of nanofibrous films with their representative SEM images were given in Fig. 2. The nanofibers of paracetamol/CD-ICs are self-standing, easy to handle and flexible. The average fiber diameter (AFD) was calculated as $775 \pm 285 \mathrm{~nm}$ for paracetamol/HP $\beta C D-I C$ NFs and $610 \pm 365 \mathrm{~nm}$ for paracetamol/SBE- $\beta$-CD-IC NFs (Table 1). Although the AFD value for both nanofibers was not very different from each other, the slight difference was possibly due to the variation in viscosity of the CD-IC solutions (Table 1). The higher viscosity causes the formation of thicker nanofibers due to the greater resistance to stretching of solution [36]. Here, the viscosity of paracetamol/HP $\beta C D-$ IC NFs is higher than paracetamol/SBE- $\beta$-CD-IC NFs, therefore, this system resulted in thicker fibers. 


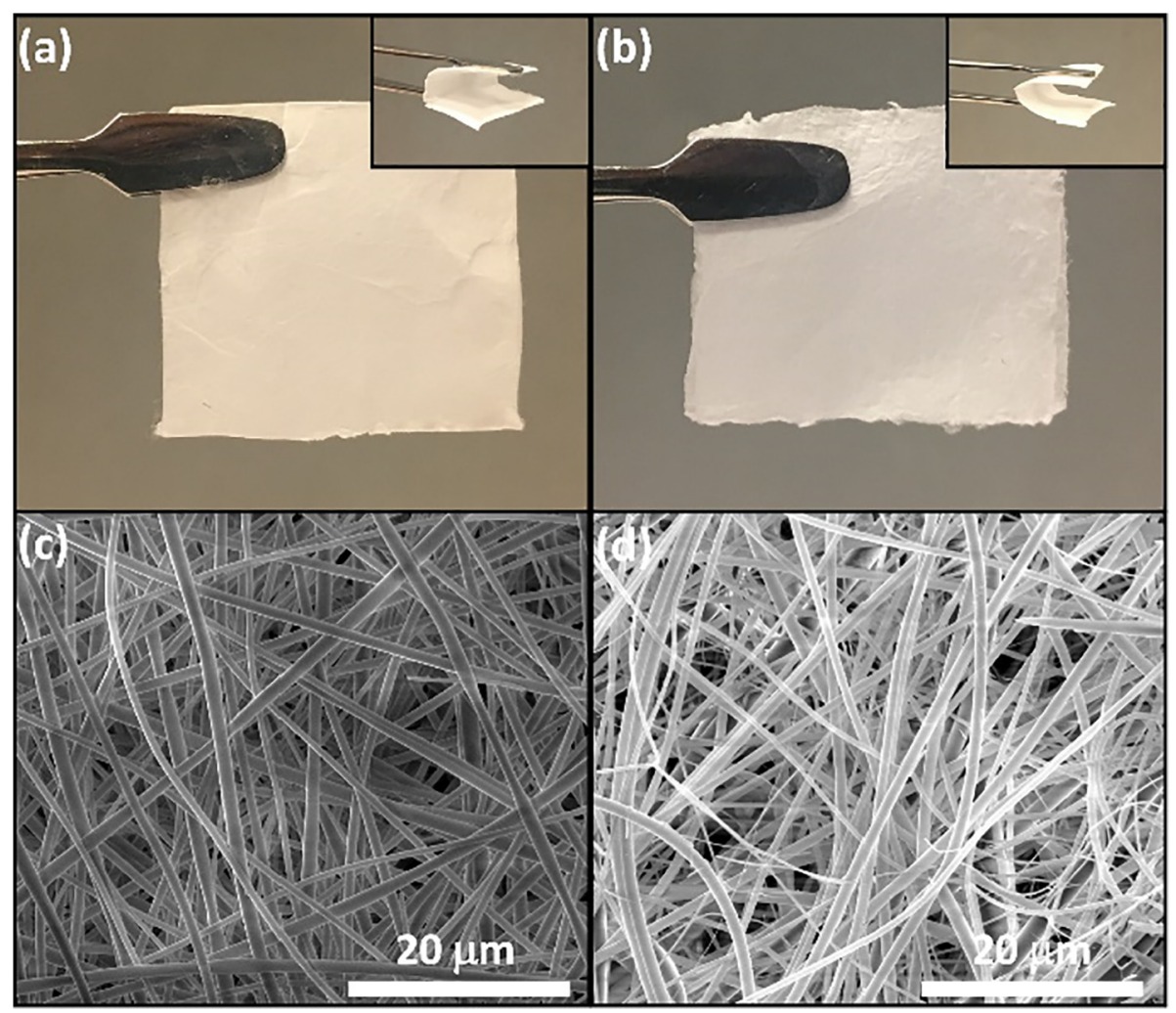

Fig. 2. The photographs of (a) paracetamol/HP $\beta C D-I C$ NFs and (b) paracetamol/SBE- $\beta$-CD-IC NFs; SEM images of electrospun (c) paracetamol/HP $\beta C D-I C$ NFs and (d) paracetamol/SBE- $\beta$-CD-IC NFs.

\subsection{The molar ratio of paracetamol/CD-ICs}

Proton nuclear magnetic resonance $\left({ }^{1} \mathrm{H}\right.$ NMR) was employed to find the molar ratio of paracetamol to CDs for paracetamol/HP $\beta$ CD-IC and paracetamol/SBE- $\beta$-CD-IC NFs. The molar ratio was calculated by integrating peaks of paracetamol (at around $6.65 \mathrm{ppm}$ ), HPßCD (at $1 \mathrm{ppm}$ ) and SBE- $\beta$-CD (at around $5 \mathrm{ppm}$ ) (Fig. 3). The molar ratio was calculated as $\sim 0.85: 1.00$ (paracetamol:CD) for paracetamol/HP $\beta C D-I C$ NFs and as $\sim 0.80: 1.00$ (paracetamol:CD) for paracetamol/SBE- $\beta$-CD-IC NFs. When compared with initial molar ratio which is $1.00: 1.00$, it is concluded that the paracetamol was mostly preserved for both CD types with similar ratios in these electrospun nanofibrous films.

\subsection{Structural characterization of paracetamol/CD-IC NFs}

FTIR is widely used characterization technique to verify the formation of inclusion complexes between CD host and guest materials and to verify presence of both guest and host molecules in their inclusion complexes. The infrared spectra of paracetamol, pure CD NFs and paracetamol/CD-ICs NFs were obtained and presented in Fig. 4. The IR spectrum of paracetamol is characterized by absorption bands at $3326 \mathrm{~cm}^{-1}$ (N-H amide stretching), $3160 \mathrm{~cm}^{-1}$ (free $-\mathrm{OH}$ stretching), $1655 \mathrm{~cm}^{-1} \quad\left(\mathrm{C}=\mathrm{O}\right.$ stretching), $\quad 1610 \mathrm{~cm}^{-1} \quad(\mathrm{C}=\mathrm{C} \quad$ stretching), $1565 \mathrm{~cm}^{-1}$ ( $\mathrm{N}-\mathrm{H}$ amide II bending), $1507 \mathrm{~cm}^{-1}$ (asymmetrical $\mathrm{C}-\mathrm{H}$ bending), $1443 \mathrm{~cm}^{-1}$ (C-C stretching), $1368-1328 \mathrm{~cm}^{-1}$ (symmetrical
$\mathrm{C}-\mathrm{H}$ bending) and $1260-1227 \mathrm{~cm}^{-1}$ (C-N stretching) [37,38]. Some peaks of paracetamol become invisible due to the extensive overlap of these bands with those of the cyclodextrins. However, there is decrease in intensity of peaks at 1565, 1507 and $1260 \mathrm{~cm}^{-1}$. Besides, the absorption peaks at $1565 \mathrm{~cm}^{-1}$ shifted to $1554 \mathrm{~cm}^{-1}$ for paracetamol/ HP $\beta C D-I C$ and to $1558 \mathrm{~cm}^{-1}$ for paracetamol/SBE- $\beta$-CD-IC and at $1507 \mathrm{~cm}^{-1}$ shifted to $1514 \mathrm{~cm}^{-1}$ for both CD-ICs. These results suggested that there is an interaction between paracetamol and CDs, and paracetamol was included into the cavity of CDs.

The crystalline behavior of paracetamol, pure CD NFs and paracetamol/CD-ICs NFs was evaluated by XRD (Fig. 5a). Paracetamol is a crystalline material showing sharp diffraction peaks appearing at $2 \theta$ $15.30^{\circ}, 18.00^{\circ}, 20.19^{\circ}, 24.17^{\circ}$ and $26.36^{\circ}[29,39]$. In the literature, there is a study in which electrospun nanofibers including paracetamol has shown amorphous character due to spreading of paracetamol molecules through nanofibrous films and these films have shown highly rapid dissolution in water [26]. In another study, formation of CD-IC of paracetamol with a modified $C D$ caused change in its crystalline structure into amorphous due to isolation of paracetamol molecules by CD-IC formation [27]. In this study, similar results with the literature has been obtained; the XRD pattern of pure CD NFs and paracetamol/ CD-ICs NFs are similar and do not exhibit any crystalline peak confirming their amorphous state. This verifies that paracetamol molecules in the paracetamol/CD-ICs were isolated from each other by the CD cavity so they cannot form crystalline aggregates. This change in

Table 1

Properties of the electrospinning solutions and the resulting nanofibers.

\begin{tabular}{|c|c|c|c|c|c|}
\hline Solutions & $\begin{array}{l}\text { Average fiber diameter } \\
(\mathrm{nm})\end{array}$ & $\begin{array}{l}\text { Fiber diameter range } \\
(\mathrm{nm})\end{array}$ & $\begin{array}{l}\text { Viscosity } \\
\text { (Pa:s) }\end{array}$ & $\begin{array}{l}\text { Conductivity } \\
\left(\mu \mathrm{S} \mathrm{cm}^{-1}\right)\end{array}$ & Morphology \\
\hline Paracetamol/HP $\beta C D-I C$ & $775 \pm 285$ & $261-1426$ & $2.37 \pm 0.23$ & $7.57 \pm 0.41$ & Bead-free nanofibers \\
\hline Paracetamol/SBE- $\beta$-CD-IC & $610 \pm 365$ & $125-1854$ & $2.06 \pm 0.12$ & $6.42 \pm 0.12$ & Bead-free nanofibers \\
\hline
\end{tabular}



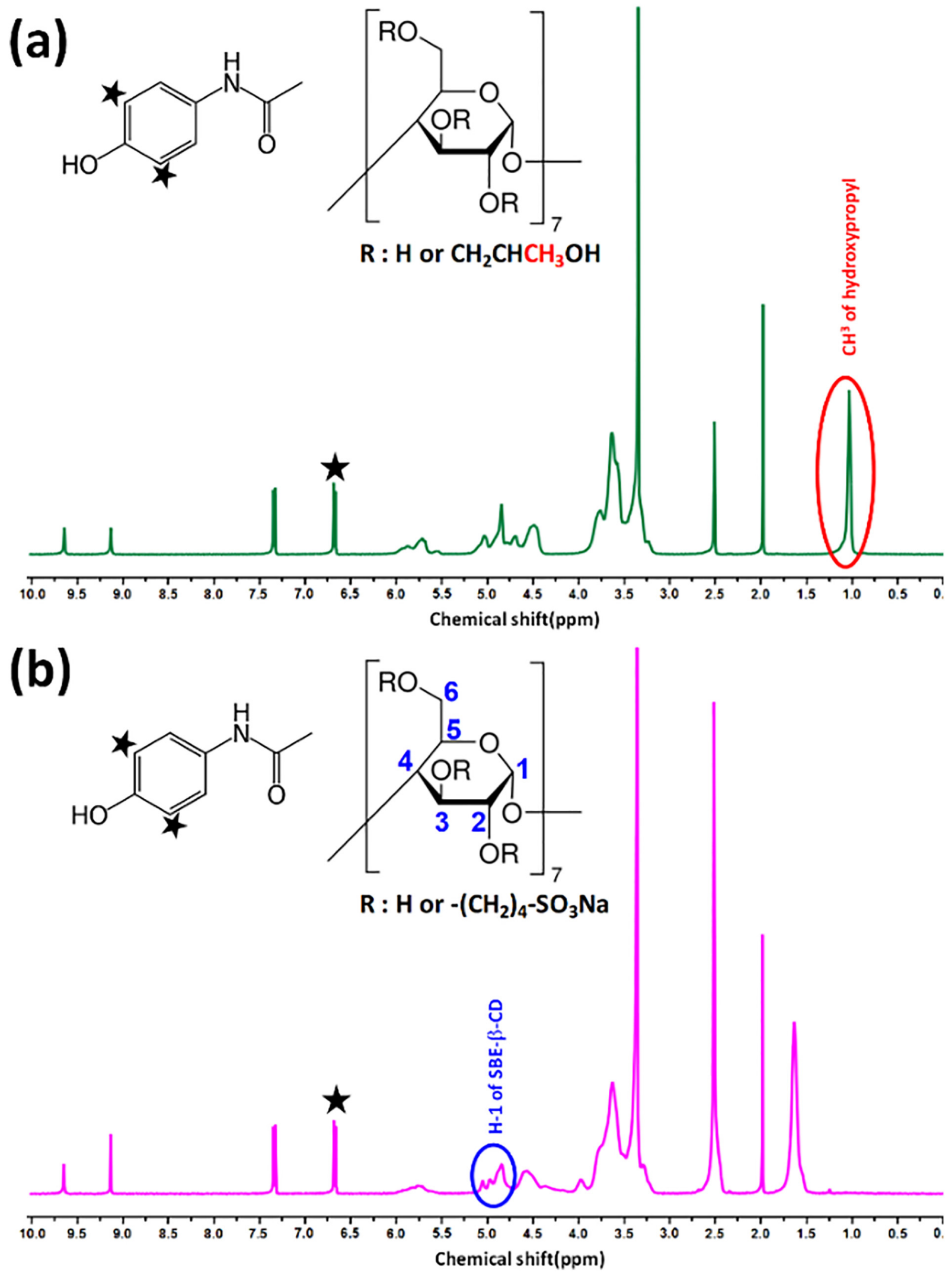

Fig. 3. The NMR spectra of (a) paracetamol/HP $\beta C D-I C$ NFs and (b) paracetamol/SBE- $\beta-C D-I C$ NFs which was dissolved in DMSO- $d_{6}$. 


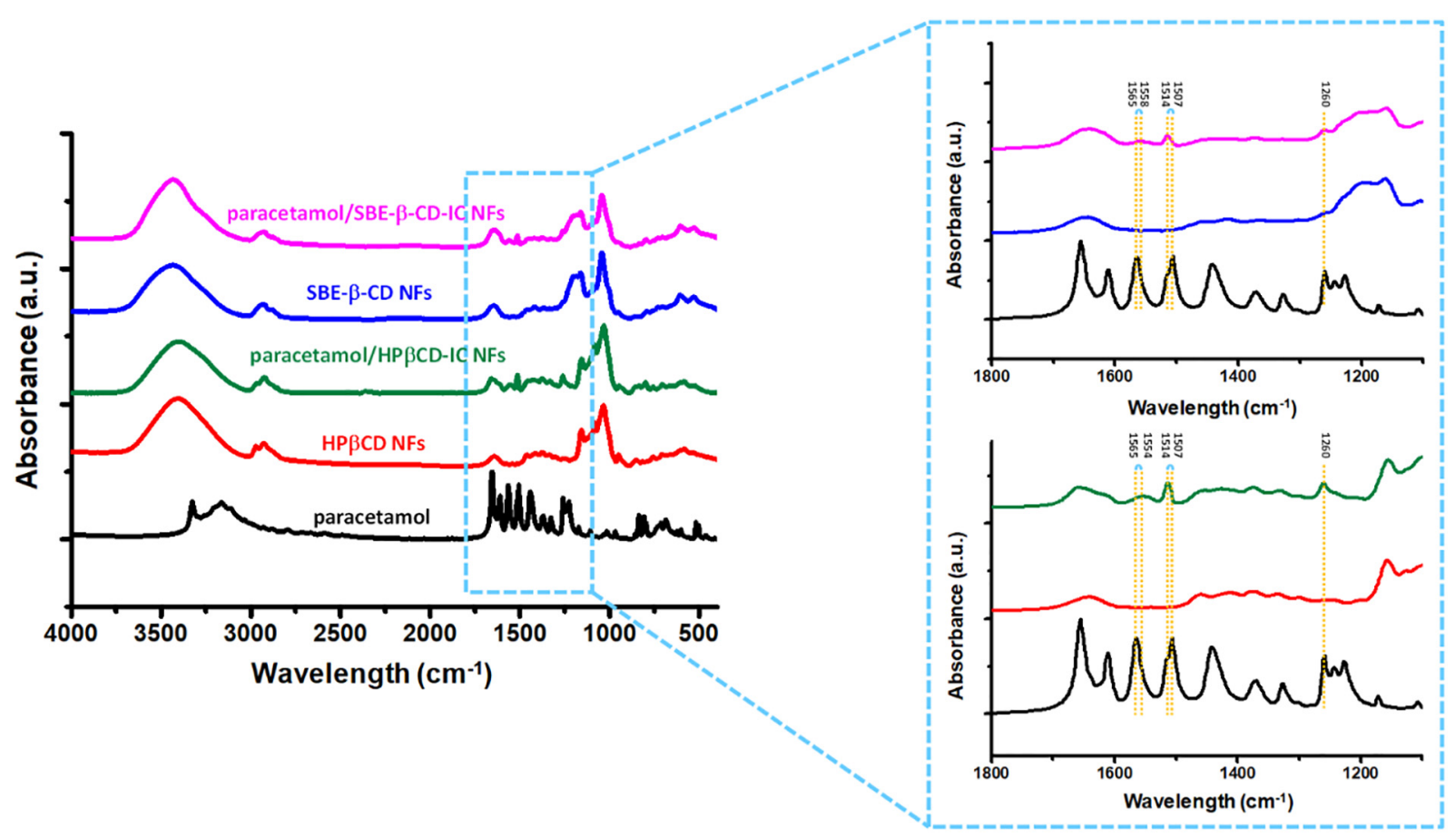

Fig. 4. The FTIR spectra of paracetamol, pure CD NFs and paracetamol/CD-IC NFs.

(a)

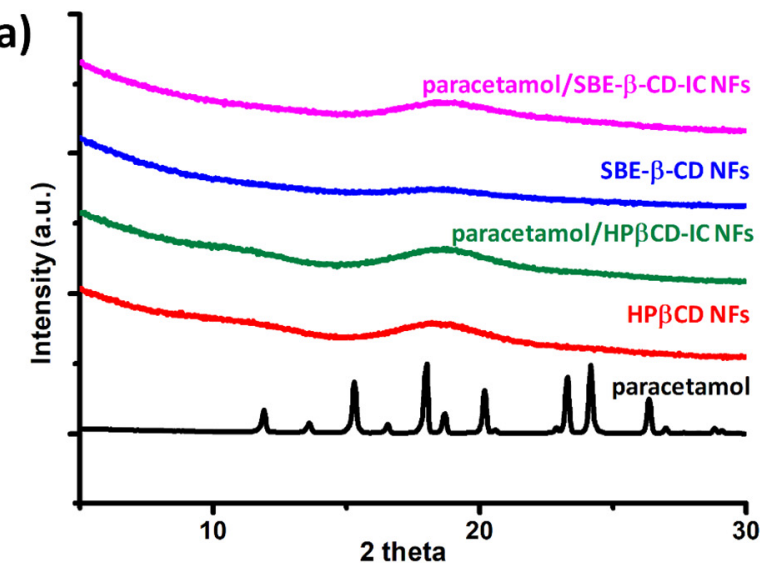

(b)

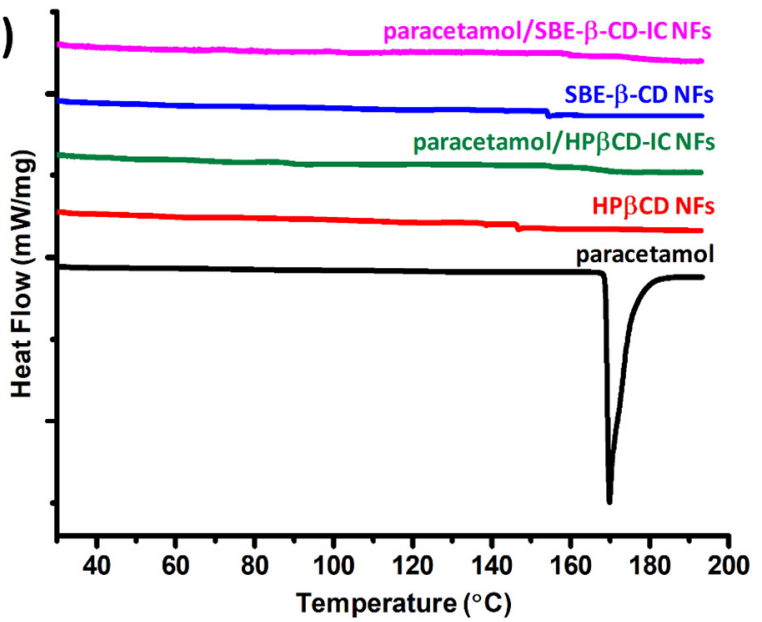

Fig. 5. (a) The XRD patterns of paracetamol, pure CD NFs and paracetamol/CDIC NFs and (b) the DSC thermogram of paracetamol, pure CD NFs and paracetamol/CD-IC NFs. crystalline structure of drug results in solubility increment since drugs in crystalline forms are more stable that decreases their solubility [40].

\subsection{Thermal characterization of paracetamol/CD-IC NFs}

DSC technique is widely used to confirm the inclusion complexation between $\mathrm{CD}$ and guest molecules. When guest molecules form inclusion complexes with CDs, their melting, boiling or sublimation points disappear or shift to higher temperatures. Fig. 5b shows the DSC thermogram of paracetamol, pure CD NFs and paracetamol/CD-ICs NFs. The DSC thermogram of paracetamol displayed a sharp endothermic peak at $169.8^{\circ} \mathrm{C}$, which corresponds to its melting point. For both pure CD NFs, there was no endothermic or exothermic peak observed. For the thermograms of paracetamol/CD-ICs NFs, the endothermic melting peak of paracetamol disappeared since paracetamol forms the molecular inclusion in the cavity of both CDs and become amorphous. These results confirm the formation of inclusion complexes and agree with the data of XRD.

Thermogravimetric analysis (TGA) was performed to identify the effects of inclusion complex formation on thermal decomposition of paracetamol (Fig. 6). The CD samples and CD-IC samples has weight losses below $100^{\circ} \mathrm{C}$ due to water loss. For pure HP $\beta C D$ NFs, the degradation started at $290^{\circ} \mathrm{C}$ while for the paracetamol/HP $\beta C D-I C ~ N F s$, there is a step before $290^{\circ} \mathrm{C}$ starting at $276{ }^{\circ} \mathrm{C}$ which belongs to paracetamol. For pure SBE- $\beta-C D$ NFs, there is mainly two step weight lost and it started at $255^{\circ} \mathrm{C}$, while for paracetamol/SBE- $\beta$-CD-IC NFs, there is an extra step at $200{ }^{\circ} \mathrm{C}$ corresponding to paracetamol degradation. These results mean that the thermal decomposition of paracetamol which started at $172{ }^{\circ} \mathrm{C}$ shifted to higher temperature, $\sim 275^{\circ} \mathrm{C}$ for paracetamol/HP $\beta C D-I C$ NFs and $\sim 200{ }^{\circ} \mathrm{C}$ for paracetamol/SBE- $\beta$-CD-IC NFs due to inclusion complex formation with CDs.

\subsection{Dissolution behavior of paracetamol/CD-IC NFs}

Dissolution behavior of paracetamol/CD-IC NFs was examined with two different methods. In the first method, the distilled water was added to the nanofibers. In the other method, CD-IC NFs of paracetamol was exposed to distilled water soaked absorbent paper [41]. For both 

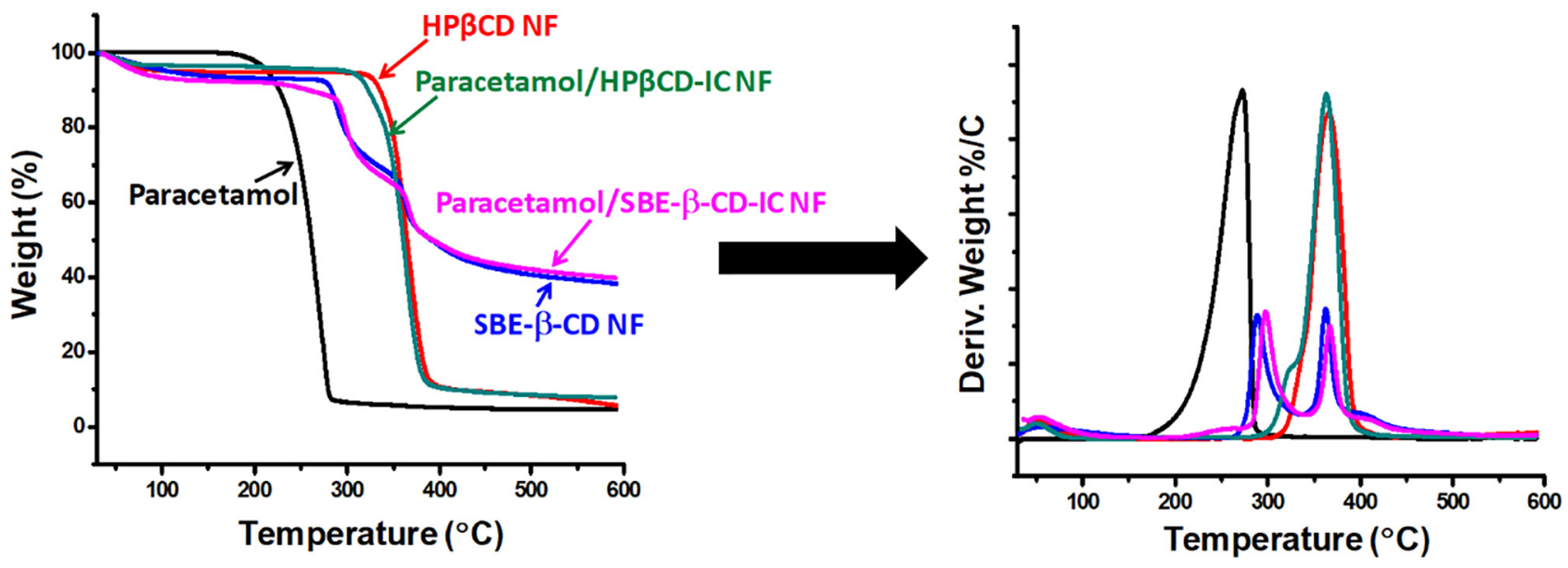

Fig. 6. The TGA thermograms and derivatives of paracetamol, pure CD NFs and paracetamol/CD-IC NFs.

methods, it was seen that paracetamol/HPßCD-IC NFs and paracetamol/SBE- $\beta$-CD-IC NFs was dissolved instantly when they are in contact with water (Fig. 7, Video S1 and Video S2). This high rate is probably due to high surface area of nanofibrous structure and solubility increase mediated by CD-IC formation.

\section{Conclusion}

In this study, free-standing, easy to handle nanofibrous films of paracetamol/HP $\beta C D-I C$ and paracetamol/SBE- $\beta$-CD-IC were successfully obtained by electrospinning. The obtained molar ratios from ${ }^{1} \mathrm{H}$ NMR analysis showed that the paracetamol molecules were mostly preserved during fabrication of nanofibrous films. Presence of paracetamol in nanofibrous films and formation of inclusion complexes were confirmed by using different characterization techniques including ${ }^{1} \mathrm{H}$ NMR, FTIR, XRD, DSC. Besides, thermal stability of paracetamol became higher after CD-IC formation. The most remarkable property of these nanofibrous films is their ultra-fast dissolving character. Two different methods were applied to analyze the dissolution behavior of nanofibrous films and films dissolved less than even a second when they exposed to water. In the light of these results, the bioavailability of paracetamol is expected to be enhanced due to enhancement in solubility and stability. It is also expected that undesirable taste of paracetamol was masked by CD-IC formation without any
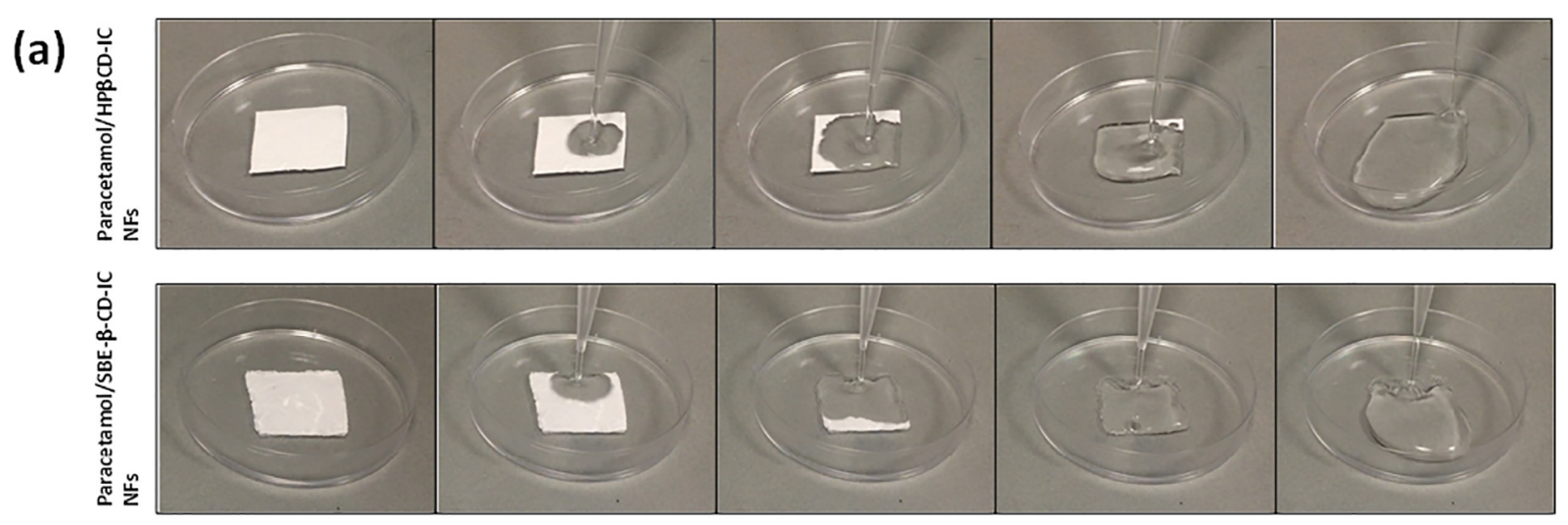

(b)
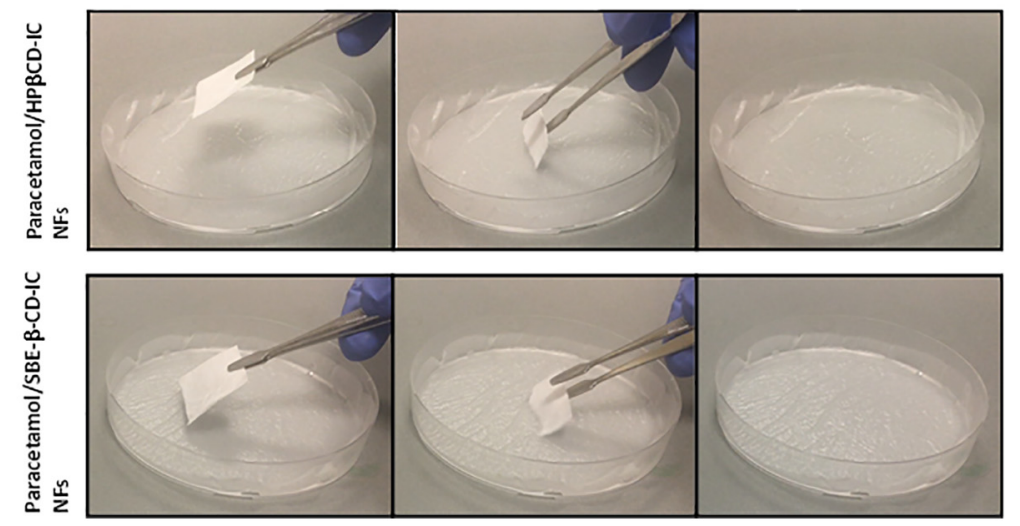

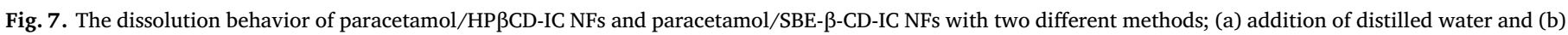

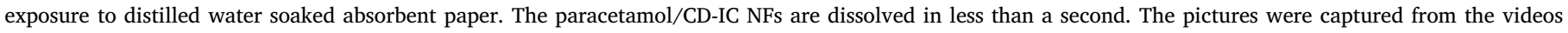
which were given as Video S1 and Video S2. 
necessity to additional flavoring agent. In brief, CDs are well known for their non-covalent inclusion complexes with drug molecules in which such inclusion complexation remarkably enhances the drug solubility. The use of CD-ICs is a promising approach to improve aqueous solubility and dissolution rate of the drug molecules. Here, both commercially available CD types (HP $\beta C D$ and SBE- $\beta-C D$ ) which are already been used in drug formulations were chosen to prepare nanofibrous films of paracetamol/HP $\beta$ CD-IC and paracetamol/SBE- $\beta$-CD-IC. The promising results obtained from this study regard to improved properties and fast-dissolving of paracetamol due to paracetamol/CD-IC nanofibrous structure may be quite interesting for the pharmaceutical applications.

The dissolution behavior of paracetamol/HP $\beta C D-I C$ NFs and paracetamol/SBE- $\beta$-CD-IC NFs with two different methods (Video S1 and Video S2). Supplementary data to this article can be found online at https://doi.org/10.1016/j.apsusc.2019.06.220.

\section{Funding}

This research received no external funding.

\section{Acknowledgments}

\section{Z. I. Y. thanks to TUBITAK-BIDEB for the PhD scholarship.}

\section{References}

[1] F.J. Otero-Espinar, J.J. Torres-Labandeira, C. Alvarez-Lorenzo, J. Blanco-Mendez Cyclodextrins in drug delivery systems, J. Drug Delivery Sci. Technol. 20 (2010) 289-301.

[2] C. Muankaew, T. Loftsson, Cyclodextrin-based formulations: a non-invasive platform for targeted drug delivery, Basic Clin. Pharmacol. 122 (2018) 46-55.

[3] P. Saokham, C. Muankaew, P. Jansook, T. Loftsson, Solubility of cyclodextrins and drug/cyclodextrin complexes, Molecules 23 (2018).

[4] M.E. Davis, M.E. Brewster, Cyclodextrin-based pharmaceutics: past, present and future, Nat. Rev. Drug Discov. 3 (2004) 1023-1035.

[5] E.M.M. Del Valle, Cyclodextrins and their uses: a review, Process Biochem. 39 (2004) 1033-1046.

[6] S.V. Kurkov, T. Loftsson, Cyclodextrins, Int. J. Pharm. 453 (2013) 167-180.

[7] M. Dinari, F. Bina, T. Khayamian, Poly(vinyl alcohol)-based electrospun nanofibers for the sustained release of celecoxib: characterization and evaluation of drug release mechanism, Polym. Compos. 39 (2018) E221-E227.

[8] T. Uyar, E. Kny, Electrospun Materials for Tissue Engineering and Biomedical Applications: Research, Design and Commercialization, Elsevier/Woodhead Publishing, Duxford, United Kingdom; Cambridge, MA, 2017.

[9] S. Bahrainian, M. Abbaspour, M. Kouchak, P.T. Moghadam, A review on fast dissolving systems: from tablets to nanofibers, Jundishapur J. Nat. Ph. 12 (2017).

[10] Z. Aytac, S. Ipek, I. Erol, E. Durgun, T. Uyar, Fast-dissolving electrospun gelatin nanofibers encapsulating ciprofloxacin/cyclodextrin inclusion complex, Colloid Surface B 178 (2019) 129-136.

[11] Z. Aytac, H.S. Sen, E. Durgun, T. Uyar, Sulfisoxazole/cyclodextrin inclusion complex incorporated in electrospun hydroxypropyl cellulose nanofibers as drug delivery system, Colloid Surface B 128 (2015) 331-338.

[12] T. Hai, X. Wan, D.G. Yu, K. Wang, Y.Y. Yang, Z.P. Liu, Electrospun lipid-coated medicated nanocomposites for an improved drug sustained-release profile, Mater Design 162 (2019) 70-79.

[13] D.G. Yu, C. Yang, M. Jin, G.R. Williams, H. Zou, X. Wang, S.W.A. Bligh, Medicated Janus fibers fabricated using a Teflon-coated side-by-side spinneret, Colloid Surface B 138 (2016) 110-116.

[14] Y.Y. Yang, W.B. Li, D.G. Yu, G.H. Wang, G.R. Williams, Z. Zhang, Tunable drug release from nanofibers coated with blank cellulose acetate layers fabricated using tri-axial electrospinning, Carbohydr. Polym. 203 (2019) 228-237.

[15] D.G. Yu, J.J. Li, G.R. Williams, M. Zhao, Electrospun amorphous solid dispersions of poorly water-soluble drugs: a review, J. Control. Release 292 (2018) 91-110.

[16] P. Vass, B. Demuth, A. Farkas, E. Hirsch, E. Szabo, B. Nagy, S.K. Andersen, T. Vigh, G. Verreck, I. Csontos, G. Marosi, Z.K. Nagy, Continuous alternative to freeze drying: manufacturing of cyclodextrin-based reconstitution powder from aqueous solution using scaled-up electrospinning, J. Control. Release 298 (2019) 120-127.

[17] G. Mitchell, Electrospinning: Principles, Practice and Possibilities, Royal Society of Chemistry, Cambridge, UK, 2015.

[18] A. Celebioglu, T. Uyar, Electrospinning of nanofibers from non-polymeric systems: polymer-free nanofibers from cyclodextrin derivatives, Nanoscale 4 (2012) 621-631.

[19] A. Celebioglu, T. Uyar, Electrospinning of nanofibers from non-polymeric systems: electrospun nanofibers from native cyclodextrins, J. Colloid Interface Sci. 404 (2013) 1-7.

[20] A. Celebioglu, T. Uyar, Antioxidant vitamin E/cyclodextrin inclusion complex electrospun nanofibers: enhanced water solubility, prolonged shelf life, and photostability of vitamin E, J. Agric. Food Chem. 65 (2017) 5404-5412.

[21] A. Celebioglu, Z.I. Yildiz, T. Uyar, Thymol/cyclodextrin inclusion complex nanofibrous webs: enhanced water solubility, high thermal stability and antioxidant property of thymol, Food Res. Int. 106 (2018) 280-290.

[22] A. Celebioglu, Z.I. Yildiz, T. Uyar, Fabrication of electrospun eugenol/cyclodextrin inclusion complex nanofibrous webs for enhanced antioxidant property, water solubility, and high temperature stability, J. Agric. Food Chem. 66 (2018) 457-466.

[23] Z.I. Yildiz, A. Celebioglu, M.E. Kilic, E. Durgun, T. Uyar, Menthol/cyclodextrin inclusion complex nanofibers: enhanced water solubility and high-temperature stability of menthol, J. Food Eng. 224 (2018) 27-36.

[24] Z.I. Yildiz, A. Celebioglu, T. Uyar, Polymer-free electrospun nanofibers from sul fobutyl ether(7)-beta-cyclodextrin (SBE7-beta-CD) inclusion complex with sulfisoxazole: fast-dissolving and enhanced water-solubility of sulfisoxazole, Int. J. Pharm. 531 (2017) 550-558.

[25] Z. Aytac, Z.I. Yildiz, F. Kayaci-Senirmak, T. Tekinay, T. Uyar, Electrospinning of cyclodextrin/linalool-inclusion complex nanofibers: fast-dissolving nanofibrous web with prolonged release and antibacterial activity, Food Chem. 231 (2017) 192-201.

[26] U.E. Illangakoon, H. Gill, G.C. Shearman, M. Parhizkar, S. Mahalingam, N.P. Chatterton, G.R. Williams, Fast dissolving paracetamol/caffeine nanofibers prepared by electrospinning, Int. J. Pharm. 477 (2014) 369-379.

[27] M. El-Kemary, S. Sobhy, S. El-Daly, A. Abdel-Shafi, Inclusion of Paracetamol into beta-cyclodextrin nanocavities in solution and in the solid state, Spectrochim. Acta A 79 (2011) 1904-1908.

[28] A.S. Balte, P.K. Goyal, S.P. Gejji, Theoretical studies on the encapsulation of Paracetamol in the $\alpha, \beta$ and $\gamma$ Cyclodextrins, J. Chem. Pharm. Res. 4 (2012) 2391-2399.

[29] D.G. Yu, C. Branford-White, K. White, X.L. Li, L.M. Zhu, Dissolution improvement of electrospun nanofiber-based solid dispersions for acetaminophen, AAPS PharmSciTech 11 (2010) 809-817.

[30] M. Hamori, K. Nagano, S. Kakimoto, K. Naruhashi, A. Kiriyama, A. Nishimura, N. Shibata, Preparation and pharmaceutical evaluation of acetaminophen nanofiber tablets: application of a solvent-based electrospinning method for tableting, Biomed. Pharmacother. 78 (2016) 14-22.

[31] H.S. Peng, S.B. Zhou, T. Guo, Y.S. Li, X.H. Li, J.X. Wang, J. Weng, In vitro degradation and release profiles for electrospun polymeric fibers containing paracetanol, Colloid Surface B 66 (2008) 206-212.

[32] W.G. Cui, X.H. Li, X.L. Zhu, G. Yu, S.B. Zhou, J. Weng, Investigation of drug release and matrix degradation of electrospun poly(DL-lactide) fibers with paracetanol inoculation, Biomacromolecules 7 (2006) 1623-1629.

[33] Q. Wang, D.G. Yu, L.L. Zhang, X.K. Liu, Y.C. Deng, M. Zhao, Electrospun hypromellose-based hydrophilic composites for rapid dissolution of poorly water-soluble drug, Carbohydr. Polym. 174 (2017) 617-625.

[34] J.J. Li, Y.Y. Yang, D.G. Yu, O. Du, X.L. Yang, Fast dissolving drug delivery membrane based on the ultra-thin shell of electrospun core-shell nanofibers, Eur. J. Pharm. Sci. 122 (2018) 195-204.

[35] W.D. Huang, Y.Y. Yang, B.W. Zhao, G.Q. Liang, S.W. Liu, X.L. Liu, D.G. Yu, Fast dissolving of ferulic acid via electrospun ternary amorphous composites produced by a coaxial process, Pharmaceutics 10 (2018).

[36] S. Ramakrishna, K. Fujihara, W.E. Teo, T.C. Lim, Z. Ma, An Introduction to Electrospinning and Nanofibers, World Scientific, 2005.

[37] A. Ebrahimi, M. Saffari, F. Dehghani, T. Langrish, Incorporation of acetaminophen as an active pharmaceutical ingredient into porous lactose, Int. J. Pharm. 499 (2016) 217-227.

[38] M.K. Trivedi, S. Patil, H. Shettigar, K. Bairwa, S. Jana, Effect of biofield treatment on spectral properties of paracetamol and piroxicam, Chem. Sci. J. 6 (2015).

[39] M. Al-Remawi, A.M.A. Ali, A. Khames, M. Hamaidi, Meloxicam-paracetamol binary solid dispersion systems with enhanced solubility and dissolution rate:preparation, characterization, and in vivo evaluation, J. Pharm. Innov. 12 (2017) 206-215.

[40] N.J. Babu, A. Nangia, Solubility advantage of amorphous drugs and pharmaceutical cocrystals, Cryst. Growth Des. 11 (2011) 2662-2679.

[41] X.Q. Li, M.A. Kanjwal, L. Lin, I.S. Chronakis, Electrospun polyvinyl-alcohol nanofibers as oral fast-dissolving delivery system of caffeine and riboflavin, Colloid Surface B 103 (2013) 182-188. 